\title{
Reconnection and Disconnection: Observations of Suprathermal Electron Heat Flux Dropouts
}

\author{
Eileen Chollet*, Ruth Skoug ${ }^{\dagger}$, John Steinberg ${ }^{\dagger}$, Nancy Crooker** and Joe Giacalone ${ }^{\ddagger}$ \\ * California Institute of Technology, Pasadena, CA 91125 \\ ${ }^{\dagger}$ Los Alamos National Laboratory, Los Alamos, NM 87545 \\ ** Boston University, Boston, MA 02215 \\ †niversity of Arizona, Tucson, AZ 85721
}

\begin{abstract}
.
Suprathermal electron heat flux dropouts (HFD) serve as a sensitive test of the magnetic topology of the inner heliosphere. Since the heat flux electron strahl always flows away from the Sun, a heat flux dropout should indicate either that the magnetic field line is completely disconnected from the Sun or that the heat flux strahl is scattered into other pitch angles. We present observations of two suprathermal electron heat flux dropout events observed by the Advanced Composition Explorer (ACE) spacecraft which occur simultaneously with impulsive energetic ion events. Since suprathermal electrons encompass the same velocity range as ions with energies of a few $\mathrm{MeV} /$ nucleon, the similarities and differences between them as observed at $1 \mathrm{AU}$ probes the sources and transport of these two species. We compare the two events to show the difference between the signatures of a simple disconnection and a more complicated reconnection scenario. Comparing suprathermal electron modulations with energetic ion modulations is a powerful technique for determining the magnetic topology between particle injection at the Sun and observation at $1 \mathrm{AU}$.
\end{abstract}

Keywords: Particle emission, Solar Wind, Solar activity

PACS: 96.60.Vg, 96.60.Q-

\section{INTRODUCTION}

Suprathermal electrons carry the coronal heat flux out into the heliosphere, after being focused into a strahl with a very narrow range of pitch angle close to the Sun. However, this strahl is sometimes observed to drop out for periods of a few hours [1]. These heat flux dropouts may produced by two mechanisms, shown in Figure 1: field line disconnection (top) and interplanetary scattering (bottom). In the former case, the total electron flux summed over pitch angle drops because the magnetic field line the spacecraft is observing disconnects from the Sun above the region where the electrons are injected. In the latter case, the pitch-angle-integrated electron flux stays roughly constant, but the electrons scatter from a narrow strahl into a more isotropic distribution during a very long travel time along a long field line [2].

The ACE spacecraft [3] at Earth's L1 point observes the solar wind constantly, including energetic ions with ULEIS [4] and suprathermal electrons with SWEPAM [5]). Because these two species have significantly overlapping speed ranges but very different scattering properties, they can jointly be used to distinguish between long field lines and disconnections. A field line which has disconnected between the ion injection and the observer should produce a dropout in the ions, while the ions' long scattering mean free path [6] means they should persist even when the electron strahl is scattered into isotropy.

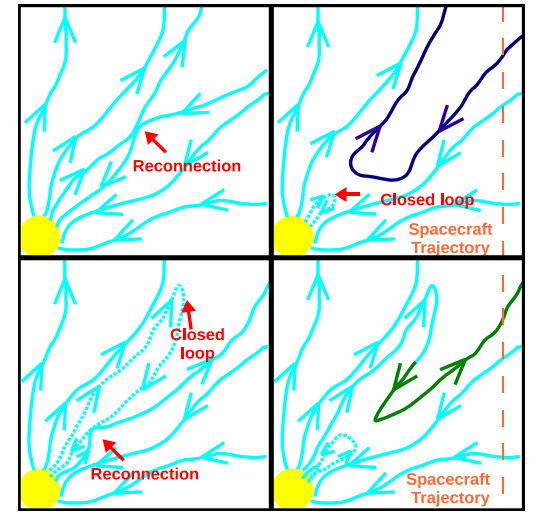

FIGURE 1. Cartoon of the possible field line configurations in heat flux dropouts.

In the following two sections, we present case studies of both types of heat flux dropout, and we demonstrate how even very complicated reconnection scenarios can be studied using energetic particles.

\section{DISCONNECTION: 28 APRIL 2000}

Simultaneous drops in ions and electrons in an event on 28 April 2000 suggest the magnetic field lines briefly disconnect from the Sun. Figures 2, 3 and 4 show the 


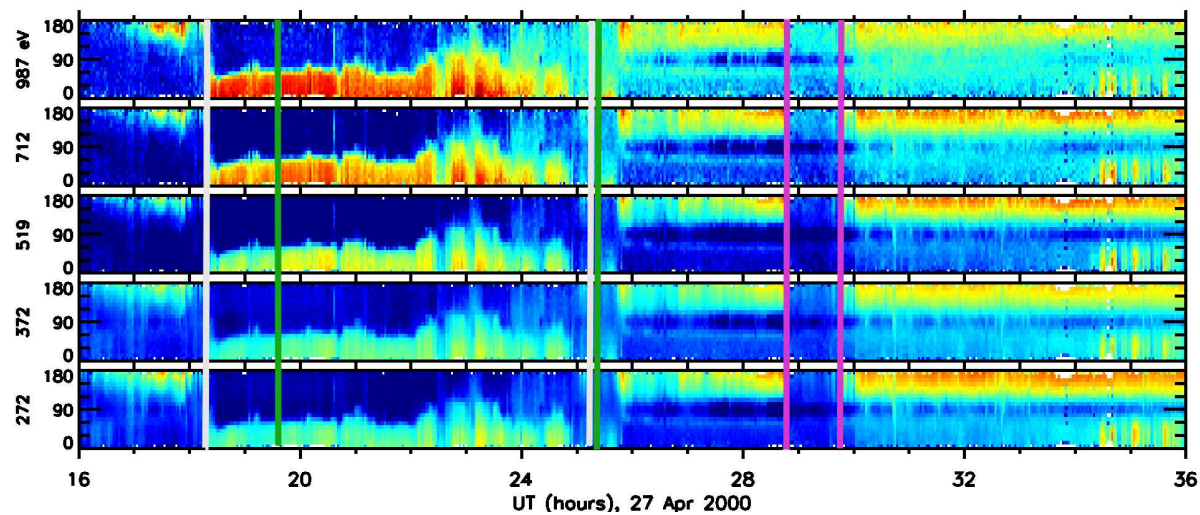

FIGURE 2. Electron pitch angle intensities in 5 SWEPAM energy bins for the 28 April 2000 event, from DOY 118.7 to DOY 119.5. The electron burst from the impulsive event begins at 16:00 UT on 27 April and persists until 1:00 UT on 28 April.

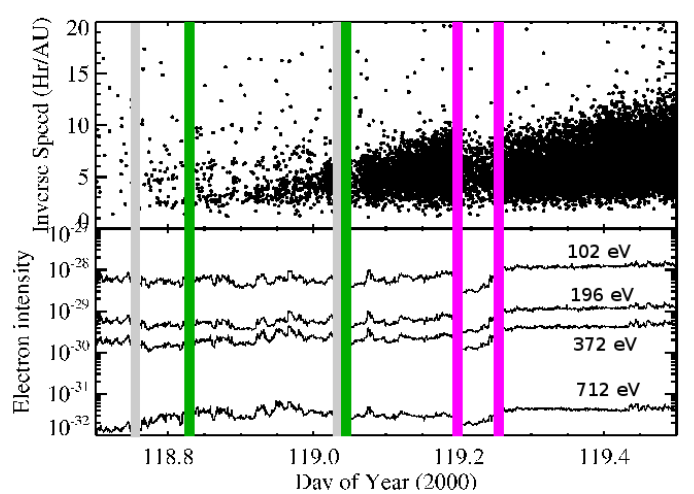

FIGURE 3. In the top panel, ULEIS ions for the 28 April 2000 event, with each dot representing a hit on the detector. The velocity dispersion is produced by the particles being injected simultaneously and having different travel times to 1 AU. The species carbon through iron are included here. In the bottom panel, the pitch-angle-integrated electron intensity in four energy channels.

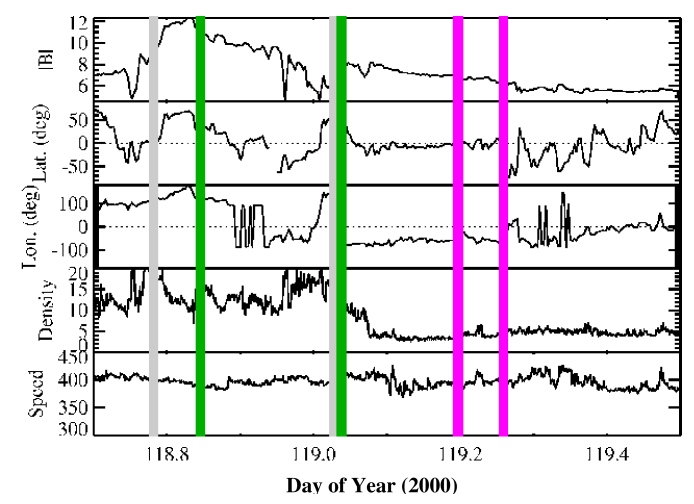

FIGURE 4. The magnetic field magnitude, magnetic latitude and longitude, solar wind density and speed for 27-28 April 2000 . electron pitch angle, ion and pitch-angle-integrated electron, and solar wind and magnetic field data for an impulsive event on 27-28 April 2000 (DOY 118-119). The pink vertical lines in all the plots mark the heat flux dropout, which lasts approximately an hour between 5:00 and 6:00 UT on the 29th according to the pitch angle data (Figure 2). During the heat flux dropout, the ions (Figure 3 , top) clearly show a dispersionless dropout. Though the edges of the ion dropout are not as sharp as those in the pitch angle plot because some ions scatter onto the unfilled field line, in the middle of the ion dropout the ion intensity gets close to the background level. At the same time, the pitch-angle-integrated electron strahl intensity (Figure 3, bottom) drops by roughly a factor of 3. In the early part of the energetic particle event, the pitch angle plots show that the strahl direction relative to the magnetic field switches twice, marked by gray vertical lines on each of the plots. Since the magnetic field polarity switches at at roughly the same time as the strahl (Figure 4 , green lines mark the magnetic polarity changes), the strahl polarity changes mark crossings of the heliospheric current sheet [7]. Intensity dropouts can be related to connection changes to different parts of Sun [8], but the lack of any obvious change in the solar wind conditions at the time of the heat flux dropout suggests that this process is local. In this case, the dropout is most likely a disconnection, with the field line completely detached from the Sun.

\section{LONG FIELD LINES: 28 DECEMBER 2000}

In contrast with the April event, the 28 December 2000 event has an HFD related to long field lines, where electrons are scattered into isotropy. Figures 5, 6 and 7 show the electron pitch angle, ion and pitch-angle- 


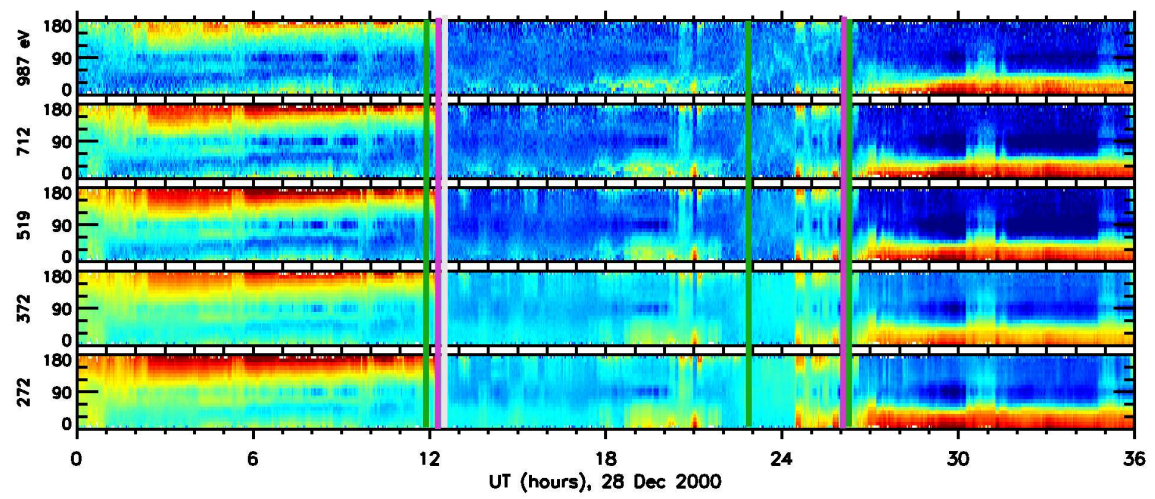

FIGURE 5. Electron pitch angle intensities in 5 SWEPAM energy bins for the 28 December 2000 event, from DOY 363.0 to DOY 364.5. The electron burst from the impulsive event begins at 0:00 UT 28 December and persists until 12:00 UT.

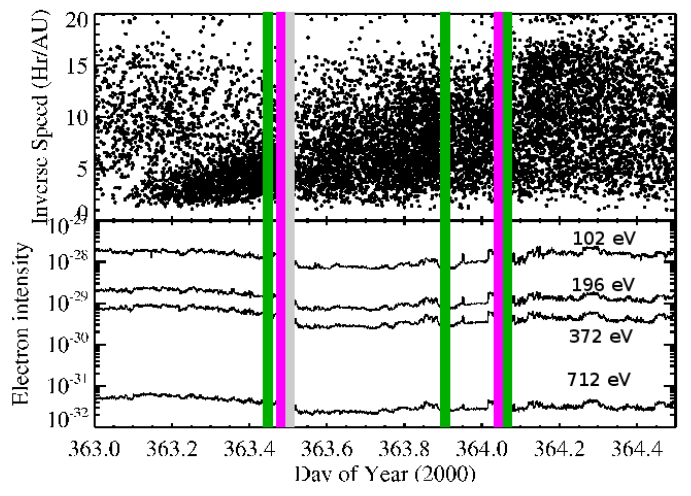

FIGURE 6. In the top panel, ULEIS ions for the 28 December 2000 event, with each dot representing a hit on the detector. The velocity dispersion is produced by the particles being injected simultaneously and having different travel times to 1 $\mathrm{AU}$. The species carbon through iron are included here. In the bottom panel, the pitch-angle-integrated electron intensity in four energy channels.

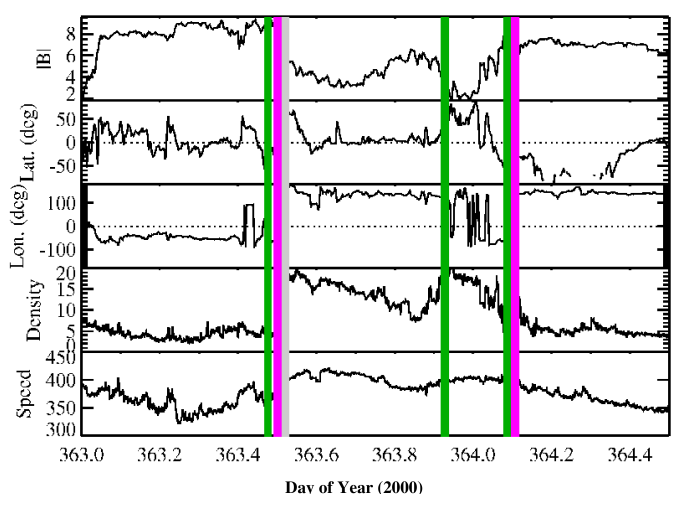

FIGURE 7. The magnetic field magnitude, magnetic latitude and longitude, solar wind density and speed for 28-29 December 2000 . integrated electron, and solar wind and magnetic field data for this event. The color coding of the vertical lines is the same as the previous case study, with pink lines marking the heat flux dropout, a gray line marking where the electron polarity switches and the green lines marking magnetic field polarity changes. The magnetic polarity is observed to be opposite of what would be expected for that side of the current sheet between the green lines at 20:00 UT on DOY 363 and 2:00 UT on DOY 364, signaling a magnetic field inversion like that in the lower right panel of Figure 1. Another inversion is observed on the 27th between 11:00 UT and 18:00 UT (not shown). During the heat flux dropout, the pitchangle-integrated electron intensity drops only by around $20 \%$, much less than the disconnection in the April event, and it recovers back to the level observed outside the HFD around 20:00 on DOY 363. The ion intensity shows a similar pattern, dropping slightly during the heat flux dropout with a brief recovery around 20:00.

\section{ANALYSIS}

Dropouts in intensity integrated over all pitch angles of either ions or electrons indicate that the magnetic field line that the spacecraft is observing is not connected to the particle source. Since suprathermal electrons are injected throughout the solar corona, a dropout in the angle-integrated electron intensity strongly indicates a field line that is disconnected from the Sun. However, because the ion injection region has been observed to be small, an ion intensity decrease may occur when field line footpoints simply fail to connect to the region by chance $[9,10]$. In the April event, ions and electrons with the same speeds drop out at exactly the same time, suggesting that the ion dropout occurs not because of the location of the field line footpoint, but instead because the field line is disconnected. For both to drop out, the 


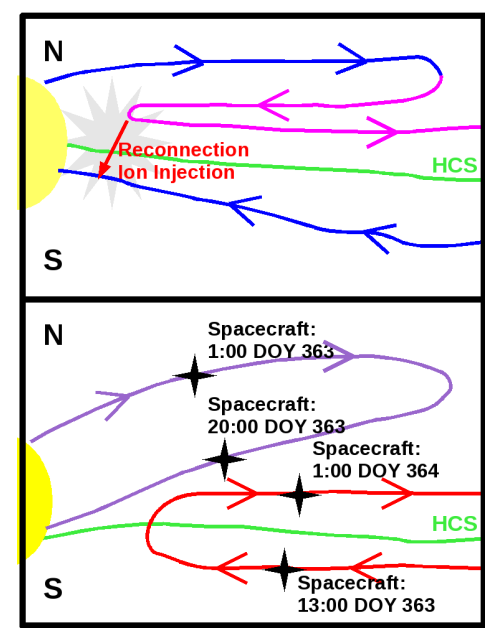

FIGURE 8. Cartoon of likely field line configurations in the 28 December 2000 event. In the upper panel, only electrons are injected onto the field lines (blue), and are scattered into isotropy on the long line (pink). In the bottom panel, ions populate red field lines, and purple field lines have a mix of ions and electrons present.

field line must have disconnected between the heights where suprathermal electrons and ions were injected and the observer. A disconnection below these heights should have no effect on the connection between the particle sources and the observer.

The December event appears to have a mix of disconnected and connected field lines produced by interchange reconnection. Figure 8 presents a possible reconnection scenario which explains the observations presented in the previous section. A field line inversion could reconnect across the heliospheric current sheet, producing a long, closed field line and a disconnected field line. The reconnection event injects ions onto both sets of field lines, but this event occurs between the electron injection region and the observer, cutting off the observer from the electron source. Closed field lines normally exhibit bidirectional strahls, since they are connected to the Sun in two places [11]. However, just like in field inversions, the length of the field line can cause an HFD in one of these strahls, producing a single narrow strahl superimposed on an isotropic distribution. Early on day 363, before the HFD begins, and during the brief recovery around 20:00, the spacecraft is connected to the closed field line and observed the single strahl plus the isotropic distribution. During the heat flux dropout, outside the brief recovery, the spacecraft is connected to the disconnected field line, so the pitch-angle-integrated electron intensity drops.

\section{CONCLUSIONS}

We have presented two case studies of events where suprathermal electron heat flux dropouts occur during energetic ion events. In the April 2000 event, a magnetic field line disconnects between the Sun and the ion injection region, producing a dropout in both the ions and the electron heat flux. In the December 2000 event, a magnetic field inversion reconnects with an open field line to produce a closed and open loop. The electron strahl is scattered into isotropy on the long portion of the closed loop, and drops out completely on the open loop. Because the reconnection injected ions onto both loops, they persist through the heat flux dropout, even though the open loop is disconnected from the Sun above the region where the suprathermal electrons are injected. Combining data for ions and electrons with the same particle speeds is a powerful technique for inferring the topology of magnetic field lines. Though the analysis performed here was simply a case study, the technique can be applied to a variety of open problems in heliophysics.

\section{REFERENCES}

1. D. J. McComas, J. T. Gosling, J. L. Phillips, S. J. Bame, J. G. Luhmann, and E. J. Smith, Sol. Phys. 94, 6907-6916 (1989).

2. C. Pagel, N. U. Crooker, D. E. Larson, S. W. Kahler, and M. J. Owens, Journal Geophys. Res. 110, CiteID A01103 (2005).

3. E. C. Stone, A. M. Frandsen, R. A. Mewaldt, E. R. Christian, D. Margolies, J. F. Ormes, and F. Snow, SSRv, 86, 1-22 (1998).

4. G. M. Mason, R. E. Gold, S. M. Krimigis, J. E. Mazur, G. B. Andrews, K. A. Daley, J. R. Dwyer, K. F. Heuerman, T. L. James, M. J. Kennedy, T. Lefevere, H. Malcolm, B. Tossman, and P. H. Walpole, SSRv 86, 409-448 (1998).

5. D. J. McComas, S. J. Bame, P. Barker, W. C. Feldman, J. L. Phillips, P. Riley, and J. W. Griffee, SSRv 86, 563-612 (1998).

6. J. W. Bieber, W. H. Matthaeus, C. W. Smith, W. Wanner, M.-B. Kallenrode, and G. Wibberenz, Astrophys. J., 420, 294-306 (1994).

7. S. Kahler, and R. P. Lin, Geophys. Res. Lett. 21, 1575-1578 (1994).

8. J. T. Gosling, R. M. Skoug, D. J. McComas, and J. E. Mazur, Astrophys. J. 614, 412-419 (2004).

9. J. Giacalone, J. R. Jokipii, and J. E. Mazur, Astrophys. J. 532, L75-L78 (2000).

10. J. E. Mazur, G. M. Mason, J. R. Dwyer, J. Giacalone, J. R. Jokipii, and E. C. Stone, Astrophys. J. 532, L79-L82 (2000).

11. J. T. Gosling, D. N. Baker, S. J. Bame, W. C. Feldman, R. D. Zwickl, E. J. Smith, Sol. Phys., 92, 8519-8535 (1987). 